Table of Contents Graphic:
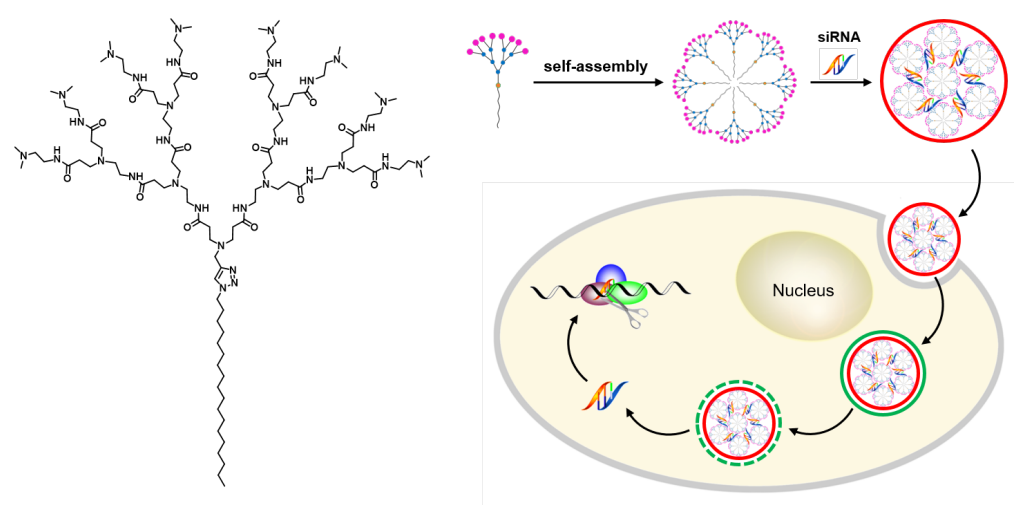

An ionizable amphiphilic dendrimer self-assembles into a supramolecular dendrimer nanosystem and delivers small interfering RNA (siRNA) with potent gene silencing and effective anticancer activity, outperforming the current gold standard delivery agent while providing a favorable safety profile. 


\title{
An ionizable supramolecular dendrimer nanosystem for effective siRNA delivery with a favorable safety profile
}

\begin{abstract}
Gene therapy using small interfering RNA (siRNA) is emerging as a novel therapeutic approach to treat various diseases. However, safe and efficient siRNA delivery still constitutes the major obstacle for clinical implementation of siRNA therapeutics. Here we report an ionizable supramolecular dendrimer vector, formed via self-assembly of a small amphiphilic dendrimer, as an effective siRNA delivery system with favorable safety profile. By virtue of the ionizable tertiary amine terminals, the supramolecular dendrimer has a low positively charged surface potential and no notable cytotoxicity at physiological $\mathrm{pH}$. Nonetheless, this ionizable feature imparted sufficient surface charge to the supramolecular dendrimer to enable formation of a stable complex with siRNA via electrostatic interactions. The resulting siRNA/dendrimer delivery system had a surface charge that was neither neutral, thus avoiding aggregation, nor too high, thus avoiding cytotoxicity, but was sufficient for favorable cellular uptake and endosomal release of the siRNA. When tested in different cancer cell lines and patient-derived cancer organoids, this dendrimer-mediated siRNA delivery system effectively silenced the oncogenes Myc and Akt2 with a potent antiproliferative effect, outperforming the gold standard vector, Lipofectamine 2000. Therefore, this ionizable supramolecular dendrimer represents a promising vector for siRNA delivery. The concept of supramolecular dendrimer nanovectors via self-assembly is new, yet easy to implement in practice, offering a new perspective for supramolecular chemistry in biomedical applications.
\end{abstract}

\section{KEYWORDS}

siRNA delivery, non-viral vector, dendrimer, supramolecular chemistry, ionizable vector, gene silencing 


\section{$1 \quad$ Introduction}

Gene therapy based on small interfering RNA (siRNA) therapeutics is emerging as a novel and promising approach to treat diseases $[1,2]$, particularly since the breakthrough success of the first siRNA drug Patirisan that was approved in 2018 [3] and the recently approved second siRNA drug Givosiran [4]. The advantages of siRNA therapeutics is their ability to potently and specifically silence any target gene via Watson-Crick base pairing with the corresponding complementary mRNA sequence and consequential cleavage of the mRNA. However, siRNA therapeutics are unstable in acidic/basic conditions and in biofluids because these RNA molecules are vulnerable to chemical and enzymatic hydrolysis. Additionally, because of their polyanionic nature and hydrophilicity, naked siRNAs cannot easily penetrate the cell membrane to reach the cell interior. Therefore, delivery systems that shield siRNA molecules from hydrolytic decomposition, cover their dense negative charge and promote their transport across cell barriers for effective gene silencing are highly sought after, which constitutes a major challenge for clinical use $[5,6]$.

Identifying safe and effective systems for siRNA delivery is an actively studied topic. Although viral vectors are generally more efficient, growing concerns over their safety and tedious manufacturing have fostered the development of non-viral vectors which, in general, are endowed with better safety profiles, easier manufacturing procedures and more flexible safe-by-design options [5-9]. Lipids and polymeric systems are the two most extensively investigated non-viral vectors $[10,11]$. Within the latter class, dendrimers, regularly branched synthetic polymers with a ramified structure, are particularly promising by virtue of their precisely controlled composition, unique cooperative multivalence and favorable high drug loading within small nanometric volumes [12-14]. However, their translation to clinical use has been hampered because of their challenging stepwise synthesis and purification [15]. 
To overcome the synthetic challenge of dendrimers and to capitalize on the advantages of lipid and dendrimer vectors, we have recently proposed innovative non-covalent supramolecular dendrimers for siRNA delivery [16-19]. The supramolecular dendrimers can be constructed via self-assembly of small amphiphilic lipid-dendrimer conjugates through van der Waals and hydrogen bond interactions, and combine the self-assembling feature of lipid vectors with the multivalent cooperativity of dendrimer vectors for siRNA delivery (Fig. 1A). Figure 1B shows one of our first amphiphilic lipid-dendrimer conjugates, 1a, which was developed for siRNA delivery [19]. The structure of 1a consists of a long alkyl chain as the hydrophobic component and a small poly(amidoamine) (PAMAM) dendron carrying primary amine terminals as the hydrophilic entity. The dendrimer 1a self-assembles into supramolecular dendrimer nanomicelles that favorably bind to siRNA for effective delivery [20]. By conjugating arginine residues at the dendrimer terminals in $\mathbf{1 a}$, we further obtained $\mathbf{1 b}$ (Fig. 1B), which mimics arginine-rich cell penetration peptides for more effective cellular uptake and better siRNA delivery [21]. In this report, we present a new ionizable supramolecular dendrimer formed via self-assemble of the amphiphilic dendrimer 1c (Fig. 1B) which, at variance with $\mathbf{1 a}$ and $\mathbf{1 b}$, carries tertiary amine dendrimer terminals for effective siRNA delivery with a favorable safety profile. 
A

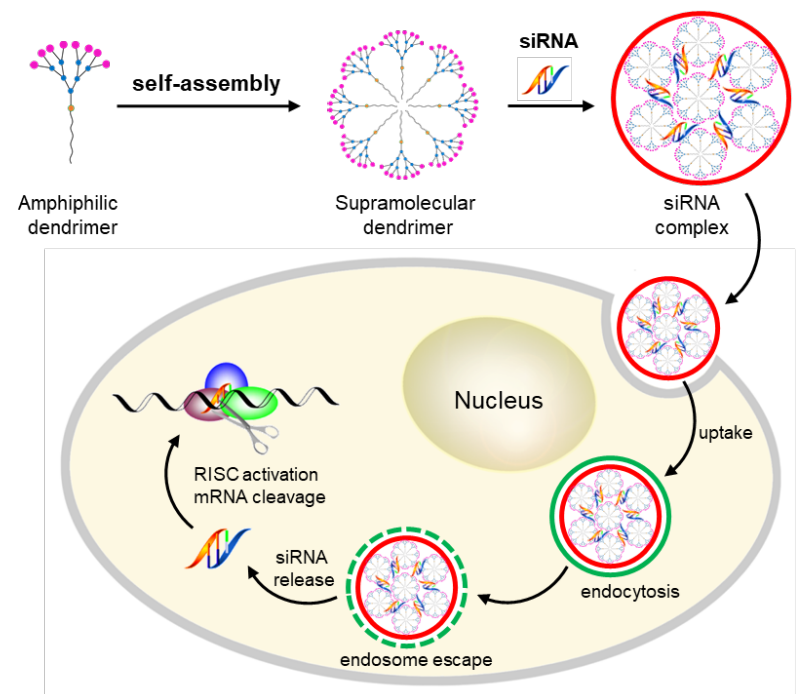

B

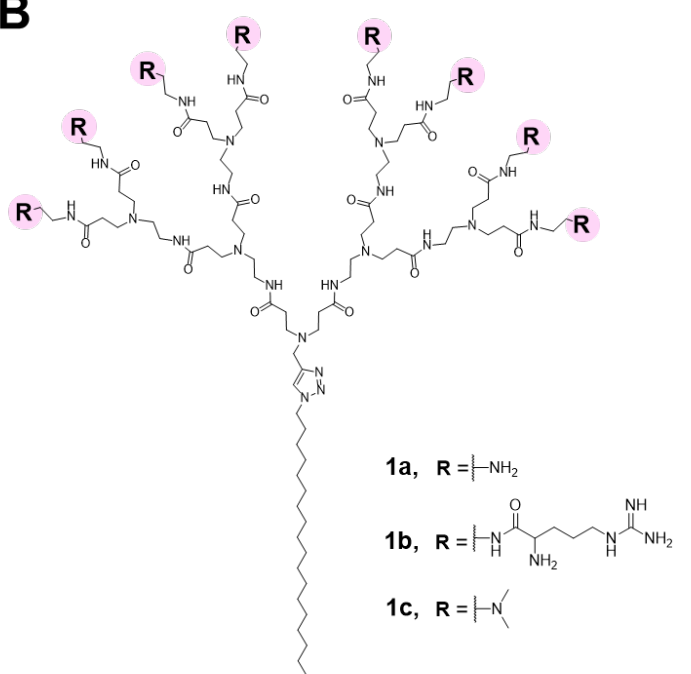

Figure 1 Schematic illustration of the supramolecular dendrimer formed via self-assembly of an amphiphilic dendrimer for siRNA delivery. (A) Cartoon presentation of the self-assembly of an amphiphilic dendrimer into a supramolecular dendrimer, which then complexes with siRNA to form a nanoparticle. This nanoparticle enters into cells via endocytosis and, upon endosomal escape, releases the siRNA into the cytosol for consequential gene silencing. (B) Chemical structures of the amphiphilic dendrimers 1a, $\mathbf{1 b}$ and $\mathbf{1 c}$ bearing primary amines, arginine residues and tertiary amines, respectively, at dendrimer terminals.

The rationale for developing the ionizable supramolecular dendrimer formed with $1 \mathbf{c}$ for siRNA delivery was based on the pKa of the tertiary amine terminals of the dendrimer surface, which will allow their protonation to generate sufficiently high positive surface charge to bind anionic siRNA through electrostatic interactions at physiological $\mathrm{pH}$, while the surface potential of the siRNA/dendrimer complexes should be low enough to prevent their clearance by the immune system yet sufficient to promote cellular uptake $[9,10,22,23]$. In addition, the ionizable tertiary amine terminals in 1c should be highly protonated in acidic endosomes, thereby inducing endosomal disruption for siRNA release. The first siRNA drug Patisiran is also based on ionizable cationic lipid nanoparticles (LNPs) for siRNA delivery. However, 
LNPs have the disadvantage of requiring specific and laborious ethanol-loading formulation techniques for preparation $[22,24]$. We demonstrate that the amphiphilic lipid-dendrimer conjugate 1c, which harbors eight tertiary amines at the surface and a further seven tertiary amines and one triazole ring in the interior, self-assembles into a supramolecular dendrimer nanosystem and acts as an ionizable cationic vector for siRNA delivery via strong siRNA binding at physiological $\mathrm{pH}$ and effective release of the siRNA within the endosomal acidic environment. Importantly, formulation of the siRNA/dendrimer complex is simple and convenient, and requires only that siRNA is mixed with $\mathbf{1 c}$ in aqueous solution. In addition, the siRNA/dendrimer complex is small and effectively protects the siRNA from enzymatic degradation. By virtue of its beneficial ionizable nature, the supramolecular dendrimer formed by $\mathbf{1 c}$ exhibited low cytotoxicity while effectively delivering siRNAs into different cancer cell lines and patient-derived cancer organoids for functional silencing of target oncogenes, leading to potent anticancer activities that outperformed the current gold standard Lipofectamine 2000 (Lipo2000). Consequently, this study offers conceptual advances in constructing supramolecular dendrimers for biomedical applications. In this study, we present the ionizable supramolecular dendrimer for siRNA delivery.

\section{$2 \quad$ Results and discussion}

We readily synthesized 1c via the amidation of the ester-terminated dendrimer precursor $\mathbf{1}$ using $N, N$-dimethylethylenediamine (Fig. 2A, Scheme S1) [25]. The amidation reaction was maintained for 5 days to ensure complete transformation of all dendrimer terminals, and the final product 1c was obtained with an excellent yield of $86 \%$ after purification via precipitation in methanol/ether followed by dialysis in water. The structural integrity and purity of $1 \mathbf{c}$ was confirmed using spectroscopic analysis with ${ }^{1} \mathrm{H}-,{ }^{13} \mathrm{C}$ - and two-dimensional (2D) NMR, as well as high-resolution mass spectrometry (Figs. S1-S3). For example, the peaks at $2.22 \mathrm{ppm}$ in the 
${ }^{1} \mathrm{H}$ NMR spectrum and at $45.3 \mathrm{ppm}$ in the ${ }^{13} \mathrm{C}$ NMR spectrum correspond to the characteristic NMR signals of the methyl groups on the tertiary amine terminals present in 1c (Fig. S1). Additionally, mass spectrometry analysis yielded the expected signals related to the molecular weight and the corresponding isotopic pattern of the triply protonated species $[\mathbf{1 c}+3 \mathrm{H}]^{3+}(\mathbf{F i g}$. S3).
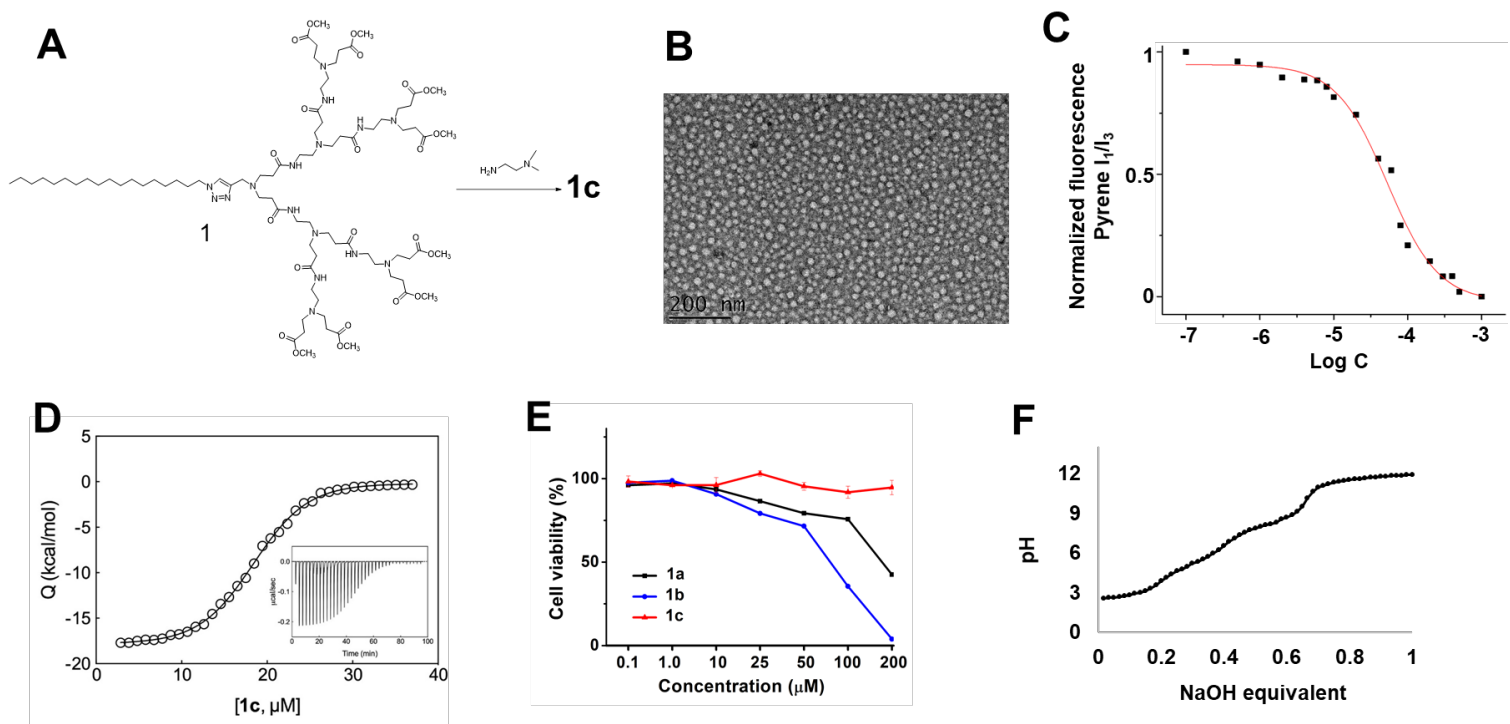

Figure 2 Synthesis, self-assembly, cytotoxicity and ionizable features of the amphiphilic dendrimer 1c. (A) Synthesis of $\mathbf{1 c}$ through amidation of the ester-terminated dendrimer 1 using $N, N$-dimethylethylenediamine. (B) A representative transmission electron microscopy (TEM) image of the supramolecular dendrimer nanomicelles formed by 1c. (C) Critical micelle concentration (CMC) of $1 \mathbf{c}$ estimated using a fluorescent spectroscopic assay with pyrene. (D) Isothermal titration calorimetry (ITC) study on the demicellization of $\mathbf{1 c}$ in PBS buffer at $\mathrm{pH}$ 7.4; measured heat (Q) as a function of $1 \mathrm{c}$ concentration. In this panel, symbols represent experimental values while the black line represents the Boltzmann data fit $\left(R^{2}=0.9983\right)$. Insert: measured heat power vs. time elapsed during the titration. (E) Absence of cytotoxicity following treatment of human embryonic kidney 293 (HEK293) cells with 1c, and comparison of the cytotoxicity of dendrimers $\mathbf{1 a}$ and $\mathbf{1 b}$. Cell viability was measured in triplicate using the PrestoBlue assay. (F) Potentiometric $\mathrm{pH}$ titration of the protonated 1c using $0.10 \mathrm{M} \mathrm{NaOH}$. 
Remarkably, $\mathbf{1 c}$ is soluble in water, with solubility similar to that of the dendrimers $\mathbf{1 a}$ and $\mathbf{1 b}$. By virtue of its amphiphilic nature, $\mathbf{1 c}$ is able to self-assemble spontaneously in water into supramolecular dendrimer micelles of $12-17 \mathrm{~nm}$ in size, as shown by transmission electron microscopic (TEM) imaging (Fig. 2B). We then evaluated the critical micelle concentration (CMC) of 1c using a fluorescent spectroscopic assay with pyrene (Fig. 2C). The CMC value of $\mathbf{1 c}$ was around $17 \mu \mathrm{M}$, which is similar to that of $\mathbf{1 a}$ [19-20]. To further monitor the process of supramolecular micelle formation by $\mathbf{1 c}$, we used isothermal titration calorimetry (ITC) (Fig. 2D and Fig. S4). The ITC results confirmed the ability of 1c to self-assemble into supramolecular dendrimer nanomicelles at a CMC value of $19 \mu \mathrm{M}$ at $\mathrm{pH} 7.4$, in accordance with the results obtained from the fluorescent spectroscopic assay. Remarkably, 1c exhibited much lower cytotoxicity than $\mathbf{1 a}$ and $\mathbf{1 b}$, particularly at higher dendrimer concentrations, as revealed by the toxicity assay on human kidney cells (HEK 293), mouse fibroblast cells (L929) and Madin-Darby canine kidney cells (MDCK) (Fig. 2E and Fig. S5). The lower toxicity of 1c might be ascribed to the distinct tertiary amine terminal groups, which generated a relatively lower surface charge for micelles formed by $1 \mathbf{c}(+23 \mathrm{mV})$ when compared with those formed by $\mathbf{1 a}(+35 \mathrm{mV})$ and $\mathbf{1 b}(+40 \mathrm{mV})$ at $\mathrm{pH} 7.4$ (Table S1). Collectively, these data confirm the spontaneous self-assembly of $\mathbf{1 c}$ to form stable supramolecular dendrimer nanomicelles with a favorable safety profile.

We next performed a potentiometric $\mathrm{pH}$ back titration of 1c, which showed continuous buffering between $\mathrm{pH} 4.0$ and 9.0, highlighting the ionizable nature of $\mathbf{1 c}$ (Fig. 2F). According to this titration profile, $50 \%$ of the amine functionalities $(8 / 16)$ of $1 \mathrm{c}$ were protonated at $\mathrm{pH}$ 7.4, likely corresponding to the eight tertiary amine terminals in 1c. At $\mathrm{pH} 5.0$, corresponding to the endosomal environment, $72 \%$ of the amine groups $(12 / 16)$ were protonated, implying that, in addition to the eight tertiary amine terminals, a further four tertiary amines located within the dendrimer interior were protonated under these conditions. Interestingly, ITC data 
repeatedly gave only small and constant endothermic peaks at pH 5.0 (Fig. S6 left), and the corresponding heat flow curve could not be used to determine any CMC value of 1c under these conditions. We reasoned that under acidic $\mathrm{pH}$ conditions 1c no longer forms stable supramolecular nanomicelles because of the growing number of positive charges populating the hydrophilic portions of the amphiphilic dendrimer. This causes stronger electrostatic repulsions within a confined volume that eventually overcome the hydrophobic attractions existing among the dendrimer hydrocarbon chains. Altogether, these results suggest that the supramolecular dendrimer formed by $\mathbf{1 c}$ will bind to siRNA at physiological $\mathrm{pH}$ while allowing endosomal release at an acidic $\mathrm{pH}$.

Effectively, the supramolecular dendrimer micelles formed by 1c at $\mathrm{pH} 7.4$ are sufficiently positively charged to allow the formation of stable complexes with the highly negatively charged siRNA molecules, thus protecting the siRNAs from degradation. We studied this siRNA/dendrimer complex formation using a gel mobility shift assay. As Figure 3A illustrates, complete retardation of siRNA migration in the gel was observed in the presence of $1 \mathrm{c}$ for $\mathrm{N} / \mathrm{P}$ ratios above 2.5 , suggesting formation of a stable siRNA/dendrimer complex. TEM imaging further showed that the siRNA/dendrimer complexes at $\mathrm{pH} 7.4$ were small and spherical, i.e., $\sim 40 \mathrm{~nm}$ in size (Fig. 3B), in line with the results obtained from dynamic light scattering (DLS) analysis (Fig. 3C). In addition, we performed mesoscale molecular simulations to investigate the interactions between siRNA and 1c, and representative siRNA/dendrimer complexes formed are presented in Figure 3D. As clearly seen in Figure 3D, the siRNA molecules are uniformly distributed and reside on the surface of the supramolecular dendrimers, highlighting the effective interaction between siRNA molecules and the terminals of the dendrimer nanomicelles. 

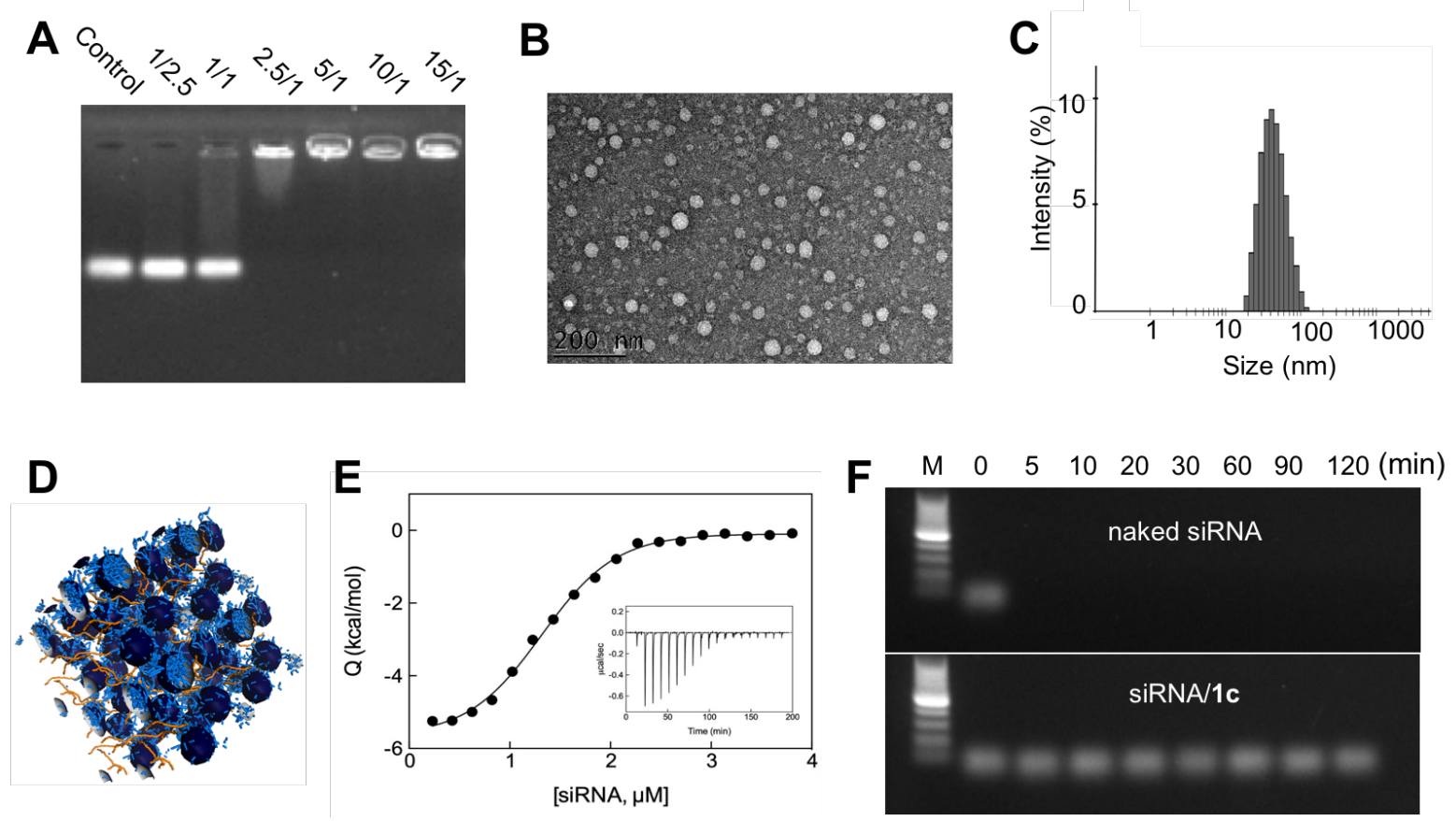

Figure 3 The supramolecular dendrimer issued from 1c formed small and stable complexes with siRNA and protected the siRNA from degradation at physiological $\mathrm{pH}$. (A) Gel retardation of siRNA with 1c at varying N/P ratios indicates the formation of stable siRNA/dendrimer complexes at N/P ratios $\geq 2.5$. (B) TEM imaging and (C) DLS analysis of the siRNA/dendrimer complex at $\mathrm{pH}$ 7.4. (D) A mesoscale molecular simulation of the siRNA/dendrimer complex (color legend: siRNA, orange sticks; amphiphilic dendrimers, light blue sticks; micelle hydrophobic cores, night blue spheres. Water and counterions are portrayed as a white field for clarity). (E) ITC data for the formation of the siRNA/dendrimer complex. Integrated ITC data (symbols) and relative fitting (solid line) using a sigmoidal model obtained from the titration of 1c with siRNA in PBS at $\mathrm{pH} 7.4\left(\mathrm{~T}=25^{\circ} \mathrm{C}\right)$. (F) The siRNA/ 1c complexes protect siRNA against RNase degradation.

We also assessed the thermodynamics of binding between siRNA and the supramolecular dendrimer formed by 1c using ITC (Fig. 3E). This revealed a negative enthalpic variation, reflecting favorable interactions between dendrimer and siRNA $(\Delta \mathrm{H}=-4.99 \mathrm{kcal} / \mathrm{mol})$, which 
indicates that the driving force behind stable siRNA/dendrimer complex formation is fundamentally electrostatic in nature. The related entropy variation upon formation of the siRNA/dendrimer complex was also favorable (i.e., $\mathrm{T} \Delta \mathrm{S}=+2.92 \mathrm{kcal} / \mathrm{mol}$ ), which originated mainly from the release of water molecules and counterions into bulk solvent upon siRNA/dendrimer binding. Overall, the resulting negative Gibbs binding free energy $(\Delta \mathrm{G}=-$ $7.91 \mathrm{kcal} / \mathrm{mol}$ ) confirms that the formation of stable siRNA/dendrimer complexes is a spontaneous and favorable thermodynamic process. Importantly, the siRNA/dendrimer complex effectively protected siRNA from enzymatic degradation (Fig. 3F). Naked siRNA was rapidly degraded within 5 min by RNase, whereas siRNA in complex with the dendrimer was not degraded readily by RNase and remained intact even after $120 \mathrm{~min}$. Taken together, our results show that the supramolecular dendrimer formed by $\mathbf{1 c}$ is sufficiently protonated and positively charged at physiological $\mathrm{pH}$ to enable interaction with siRNA to form small and stable siRNA/dendrimer complexes within which the siRNA is shielded from enzymatic degradation. These are important prerequisites for successful siRNA delivery.

Benefiting the favorable ionizable properties of the tertiary amine terminals, the siRNA complexes formed with 1c exhibited a surface potential of $+18 \mathrm{mV}$ at $\mathrm{pH} 7.4$, which is substantially lower than that of siRNA complexes formed with $\mathbf{1 a}(+31 \mathrm{mV})$ and $\mathbf{1 b}(+35 \mathrm{mV})$ (Table S1). At $+18 \mathrm{mV}$, the surface potential is neither neutral nor too high to be disadvantageous for siRNA delivery; complexes with too high surface charges may disrupt cell membranes and induce eventual toxic effects, whereas neutral complexes have limited colloidal stability and aggregate into large particles that precipitate. Importantly, this positive surface potential is also sufficient and beneficial to promote cellular uptake via favorable electrostatic interactions with the cell membrane, which is negatively charged. Notably, when the $\mathrm{pH}$ decreased from 7.4 to 5.0 , the zeta potential of the siRNA/1c complexes increased from $+18 \mathrm{mV}$ to $+46 \mathrm{mV}$, a value similar to that of dendrimer $1 \mathrm{c}$ alone without siRNA at $\mathrm{pH} 5.0$ 
$(+48 \mathrm{mV})$. This indicates that siRNA dissociates from the siRNA/dendrimer complex at $\mathrm{pH}$ 5.0, which is further supported by the results obtained from the ITC experiment examining the interaction between 1c and siRNA at $\mathrm{pH}$ 5.0. As shown in the right panel of Figure S6, no stable binding was detected between siRNA and 1c under acidic conditions, highlighting that siRNA is released readily from the dendrimer complex at $\mathrm{pH}$ 5.0. This is in sharp contrast with the data at $\mathrm{pH}$ 7.4, where siRNA and dendrimer 1c form a stable complex (Fig. 3). We further evaluated the siRNA release from its dendrimer complex using the heparin-coupled ethidium bromide assay (Fig. 4). As siRNA and dendrimer 1c form a stable complex at pH 7.4, no siRNA release was detectable using heparin at the low dose of 2 unit/mL, and only $9 \%$ siRNA release was observed when using heparin even at the high dose of 20 unit $/ \mathrm{mL}$. In contrast, more than $25 \%$ siRNA was released at $\mathrm{pH} 5.0$ even at the low heparin dose of 2.0 unit $/ \mathrm{mL}$, whereas almost all free siRNA escaped from the siRNA/1c complex at the heparin dose of 20 unit/mL. There results highlight that the siRNA/1c complex was not stable at $\mathrm{pH} 5.0$ and disassembled upon addition of heparin, hence releasing siRNA readily and easily. Collectively, these data support our rationale that, by virtue of the ionizable nature of the tertiary amine terminals within 1c, the siRNA/dendrimer complex forms easily at neutral $\mathrm{pH}$ but disassembles readily under acidic conditions. 


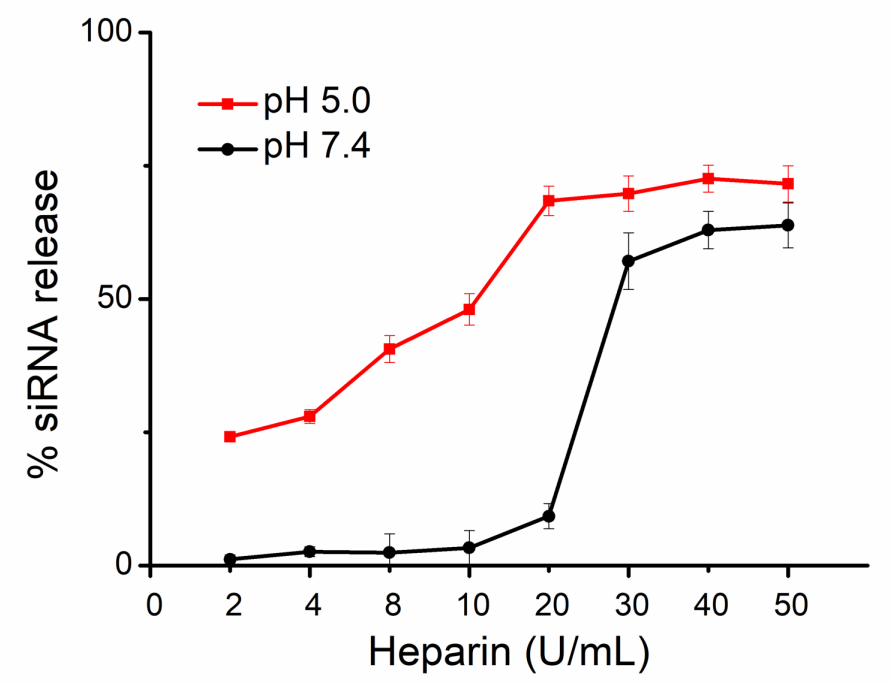

Figure 4: Release profile of siRNA from the siRNA/1c complexes at N/P 10 as a function of heparin concentration at $\mathrm{pH} 5.0$ and $\mathrm{pH} 7.4$ using heparin displacement - coupled ethidium bromide fluorescent assay.

For effective gene silencing, the siRNA/dendrimer delivery system must be able to cross cell membranes to enter cells, then escape from the acidic endosomes before releasing the siRNA cargo to reach the RNAi machinery for gene silencing. We therefore studied the cellular uptake and intracellular trafficking of the siRNA/dendrimer complexes in human pancreatic cancer Panc-1 cells using confocal fluorescence microscopy. Specifically, siRNAs were labeled with the red fluorescent tag $\mathrm{Cy} 3$, and the green fluorescent probe LysoTracker was used to trace the acidic endosomes (Fig. 5). Fluorescence imaging revealed considerable red fluorescent signals of $\mathrm{Cy} 3$ within Panc-1 cells following treatment with Cy3-siRNA/dendrimer complexes after $1 \mathrm{~h}$ (Fig. 5A), and the uptake increased as the incubation time increased (Fig. $\mathbf{5 E}$ ). In contrast, no obvious cellular uptake was observed when cells were treated with Cy3siRNA without the dendrimer even after $5 \mathrm{~h}$ incubation (data not shown). These results 
demonstrate clearly the effective internalization of siRNA mediated by the dendrimer nanovector.
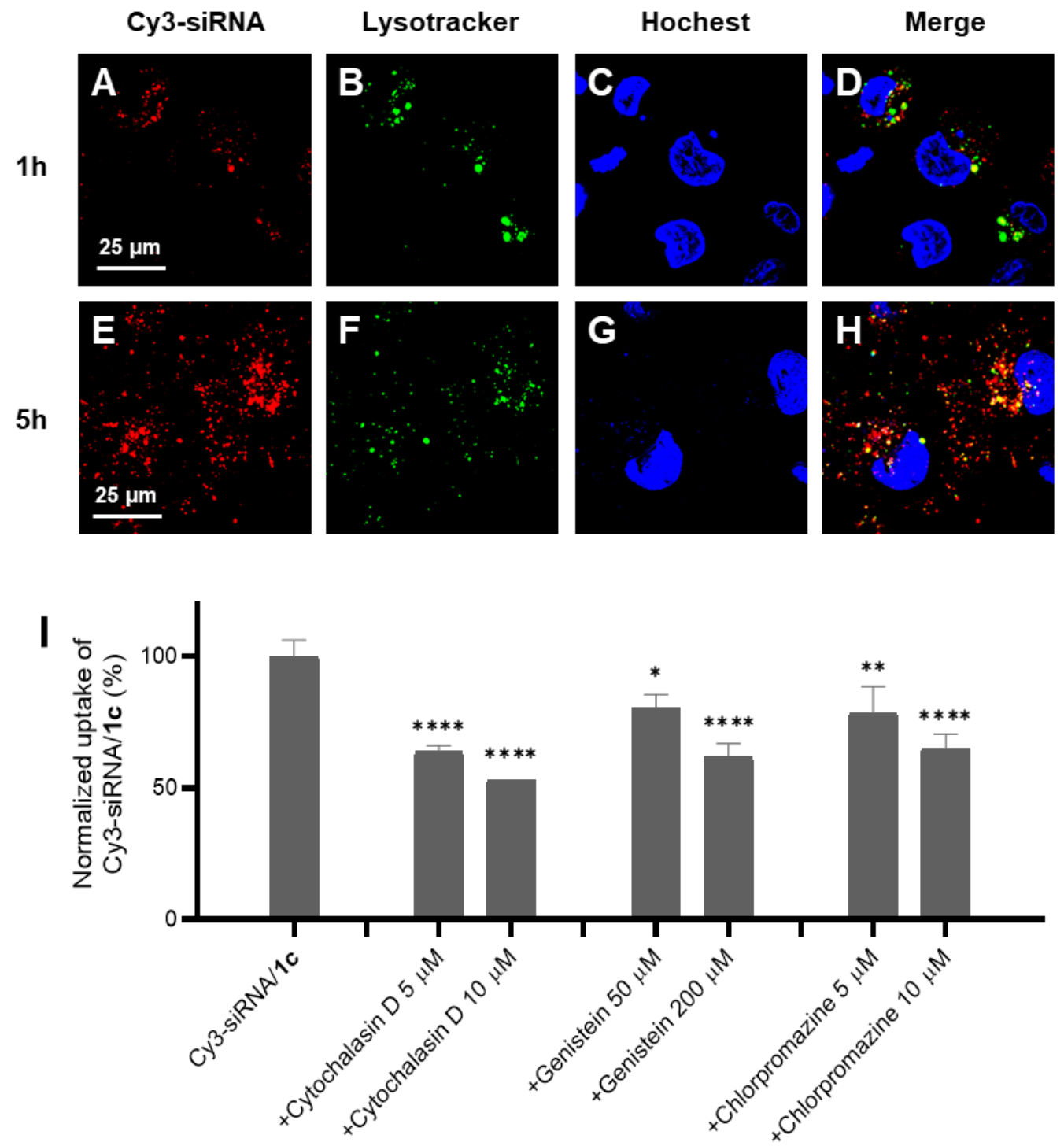

Figure 5 Cellular uptake of the siRNA/dendrimer 1c complexes in human pancreatic cancer Panc-1 cells studied using confocal microscopic imaging and FACS flow with fluorescent dye (Cy3)-labeled siRNA. Cellular uptake of the siRNA/dendrimer complexes in Panc-1 cells at 1 $\mathrm{h}(\mathrm{A}, \mathrm{B}, \mathrm{C}, \mathrm{D})$ and $5 \mathrm{~h}(\mathrm{E}, \mathrm{F}, \mathrm{G}, \mathrm{H})$ post-treatment, and (I) in the presence of inhibitors of endocytosis pathways: cytochalasin D (inhibitor of micropinocytosis), chlorpromazine (inhibitor of clathrin-mediated endocytosis) and genistein (inhibitor of caveolae-mediated 
endocytosis). (A, E) The red channel images highlight the Cy3-labeled siRNA/1c complexes; $(\mathrm{B}, \mathrm{F})$ the green channel images show the endosomes labeled with LysoTracker ${ }^{\circledR}$ Green DND26; $(\mathrm{C}, \mathrm{G})$ the blue channel images show the nuclei of Panc-1 cells stained with the fluorescent probe Hoechst 33342 staining solution; and (D, H) the merged images. (I) Cellular uptake of the siRNA/1c complex is inhibited by cytochalasin D, genistein and chlorpromazine, which are inhibitors of micropinocytosis, caveolae-mediated endocytosis and clathrin-mediated endocytosis, respectively.

We next studied the uptake mechanism for the observed internalization of the siRNA/1c complexes using specific inhibitors of endocytic pathways. Cytochalasin $D$ is a macropinocytosis inhibitor, whereas chlorpromazine and genistein are specific inhibitors of clathrin-mediated endocytosis and caveolae-mediated endocytosis, respectively. Remarkably, a significant reduction in the cell uptake of siRNA/1c complexes was observed when all these inhibitors were applied accordingly (Fig. 5I). These results highlight that micropinocytosis, clathrin-mediated endocytosis and caveolae-mediated endocytosis are all actively involved in uptake of the siRNA/1c complex.

Subsequent intracellular trafficking of the siRNA/1 complex was studied using confocal microscopic study. Co-localization of the majority of the red fluorescent signals of Cy3-labeled siRNA and the green fluorescent signals of LysoTracker after $1 \mathrm{~h}$ of treatment (Fig. 5B-5D) revealed trafficking of siRNA/1c complexes into endosomes. We further observed, after $5 \mathrm{~h}$ treatment, considerable dispersion of the red $\mathrm{Cy} 3$ fluorescent signals of siRNA and separation from the green fluorescent signals of LysoTracker (Fig. 5F-5H). These dispersed red fluorescent signals represent the effective release of Cy3-labeled siRNAs from endosomes into the cytoplasm. In combination, our results showed that the siRNA/dendrimer delivery complex 
entered cells via endocytosis and then escaped from the endosomes before effectively releasing siRNA.

Encouraged by these promising features of the siRNA/dendrimer complex, we then assessed 1c-mediated siRNA delivery and gene silencing in different human cancer cells, including pancreatic cancer Panc-1 cells, liver cancer HepG2 and Hep3B cells, and colorectal cancer HT-29 cells (Fig. 6 and Fig. S7). Two different oncogenes, Myc and Akt2, were selected as the target genes for the silencing study, because they both are promising targets for cancer treatment. Akt2 is a threonine/serine kinase frequently activated in human cancers, with essential roles in enhancing cancer cell proliferation and tumor progression $[26,27]$, whereas Myc is an undruggable proto-oncogene that is associated with the outputs of major cellular events such as cell cycle progression, cellular transformation and apoptosis [27, 28]. Treatment with the corresponding siRNAs delivered by the supramolecular dendrimer formed with 1c inhibited Myc and Akt expression effectively at both the mRNA (Fig. 6A and 6D) and protein levels (Fig. 6B and 6E), leading to potent anticancer activity (Fig. 6C and 6F). Remarkably, such RNAi efficacy was significantly higher than that obtained with the current gold standard vector Lipo2000, and no gene silencing occurred when cells were treated with 1c alone, the target siRNA alone, or the control scrambled siRNA complexed with 1c (Fig. 6 and Fig. S7). In addition, neither the dendrimer 1c alone nor the scramble siRNA/dendrimer complex showed any notable cytotoxicity (Fig. S8). These data highlight that dendrimer 1c is capable of delivering siRNA efficiently and inducing effective gene silencing without adverse cytotoxicity. 
A
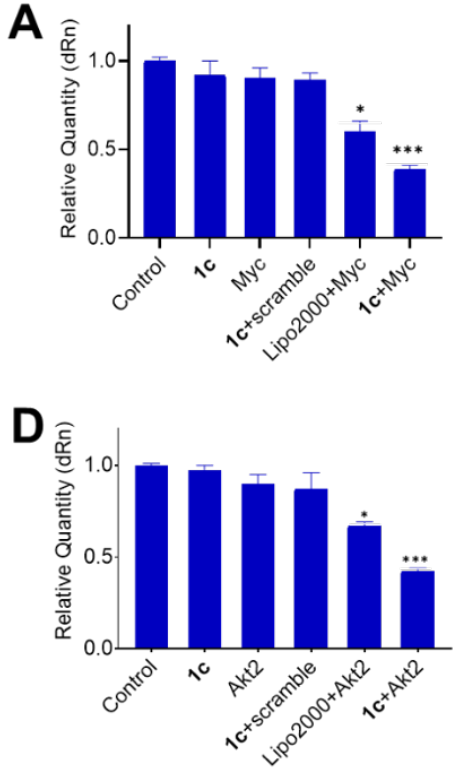

B

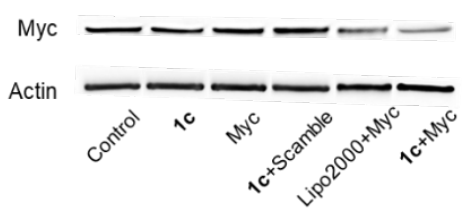

E

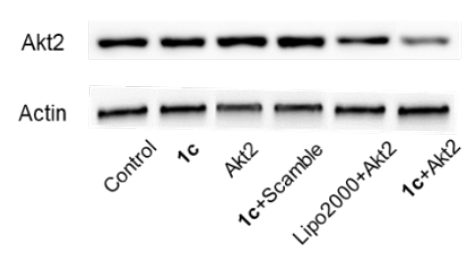

C

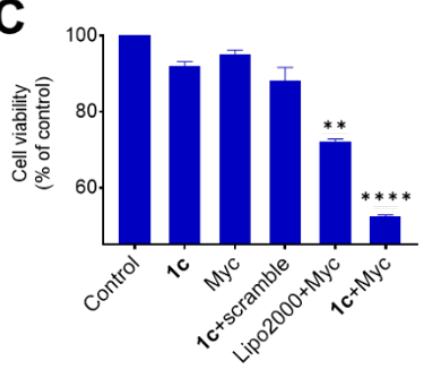

$\mathbf{F}$

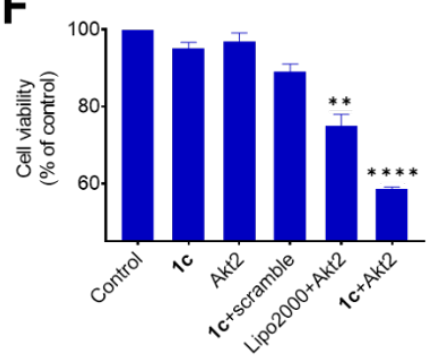

Figure 6 Supramolecular dendrimer-mediated siRNA delivery and gene silencing of the oncogenes Akt2 and Myc as well as the subsequent antiproliferative effect on human liver cancer HepG2 cells $(\mathbf{A}, \mathbf{B}, \mathbf{C})$ and human pancreatic cancer Panc-1 cells $(\mathbf{D}, \mathbf{E}, \mathbf{F})$ when compared with no treatment (control), cells treated with dendrimer 1c alone, Akt2 or Myc siRNA alone, or the scrambled siRNA/1c complex using $50 \mathrm{nM}$ siRNA at an N/P ratio of 10. The commercially available vector Lipo2000 was used as a reference control. Akt2 and Myc expression at the mRNA $(\mathbf{A}, \mathbf{D})$ and protein $(\mathbf{B}, \mathbf{E})$ levels were determined using qRT-PCR and western blotting, respectively. $(\mathbf{C}, \mathbf{F})$ Cell viability of HepG2 and Panc-1 cells upon treatment with the siRNA/1c complex was assessed using the MTT test.

We further validated the efficacy of 1c-mediated siRNA delivery and consequent anticancer activity in pancreatic cancer patient-derived organoids using siRNA targeting the oncogene Myc (Fig. 7). Notably, patient-derived organoids are emerging as robust and effective preclinical models that can be compared with traditional genetically engineered animal models and patient-derived xenograft models (PDXs) [30]. More importantly, human cancer organoids constitute reliable models for personalized medicine, as they can be used to 
accurately predict responses to therapeutic treatments [31]. Myc is an undruggable oncogene that promotes cancer cell proliferation and tumor progression [28, 29]. Therefore, using Myctargeting siRNA constitutes a promising strategy to combat pancreatic cancer [32], for which there is no effective treatment. In this study, from the PaCaOmics cohort (clinical trial registration number NCT01692873) [33, 34], we specifically selected two pancreatic cancer patient-derived-organoid models, PDAC087T and PDAC115T, as both exhibit elevated expression levels of Myc. Treatment of organoids PDAC087T and PDAC115T with 1cdelivered siRNA targeting Myc produced significant inhibition of Myc expression at both mRNA and protein levels, with subsequent inhibition of cancer cell proliferation (Fig. 7).

A

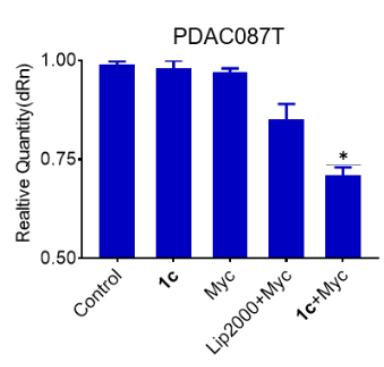

D

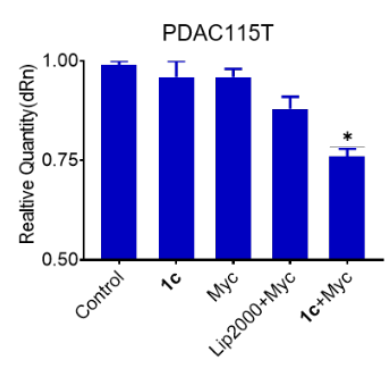

B

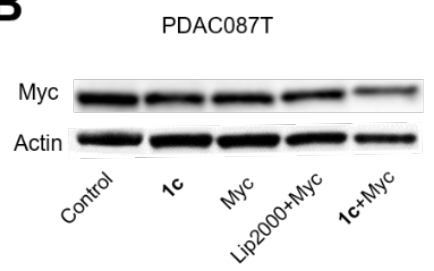

$\mathbf{E}$

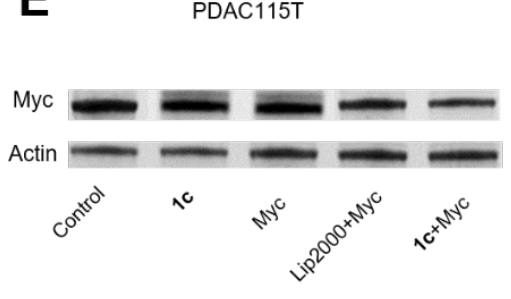

C

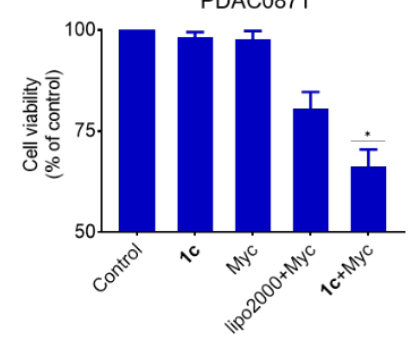

$\mathbf{F}$

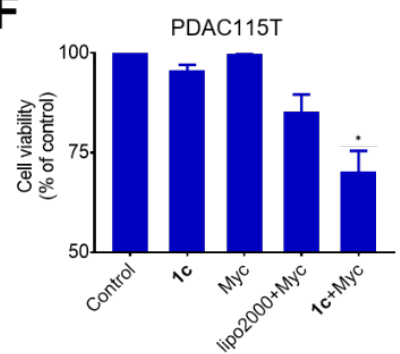

Figure 7 Delivery of Myc siRNA in patient-derived cancer organoids by the supramolecular dendrimer formed with 1c, resulting in better gene silencing and antiproliferative effect than Lipo2000. Pancreatic cancer patient-derived organoids PDAC087T and PDAC115T were treated with the Myc siRNA delivered by $1 \mathrm{c}$ using $50 \mathrm{nM}$ siRNA at an N/P ratio of 10, while the commercial vector Lipo2000 was used for comparison. Myc mRNA (A, D) and protein (B, E) levels were assessed using qRT-PCR and western blotting, respectively. (C, F) The viability of these organoids upon treatment was evaluated in triplicate using the CellTiter-Glo ${ }^{\circledR}(\mathrm{CTG})$ 
assay. Non-treated organoids and organoids treated with dendrimer 1c alone or Myc siRNA alone were used as controls.

The gene silencing efficiency of the siRNA/dendrimer 1c complex in patient-derived cancer organoids was lower than that observed for the experimental cancer cell lines. This observation can be ascribed to the intrinsic three-dimensional (3D) organization of the cancer cells within the organoids. Cell proliferation is often slower and lower in 3D organoids because of space restrictions and limitations when compared with that of cancer cells seeded on plates as two-dimensional (2D) organizations. Additionally, penetration and delivery of the siRNA/vector complexes will be less favorable within the interior of the 3D organoids because of the dense cellular organization in 3D when compared with that of cancer cells cultured on 2D plates. All these critical factors can reduce the efficiency of gene silencing in cancer organoids when compared with that of cancer cells cultured on 2D plates. Nevertheless, our supramolecular dendrimer formed by 1c showed significantly higher gene silencing and anticancer activity when compared with the gold reference Lipo2000. These results demonstrate that the supramolecular dendrimer formed by 1c is a promising system for functional siRNA delivery. In addition, siRNA-based treatment in combination with human cancer organoid models can also offer a reliable strategy for developing personalized treatment.

\section{Conclusions}

In summary, we report the ionizable supramolecular dendrimer nanovector formed by selfassembly of the amphiphilic dendrimer 1c and its effective delivery of siRNA for RNAi-based gene silencing in treating cancer. By virtue of its amphiphilic nature, 1c readily self-assembled into a supramolecular dendrimer nanovector, which acted as an efficient and safe ionizable vector for effective siRNA binding and delivery at physiological $\mathrm{pH}$ thanks to the slightly 
positive surface charge of the resulting siRNA/dendrimer complex. Contextually, it is also able to induce effective endosomal escape and subsequent cytoplasmic siRNA release at an acidic $\mathrm{pH}$, as supported by its $\mathrm{pH}$ titration profile and the experimental data for siRNA binding and endosomal release. Remarkably, the siRNA/dendrimer complexes protected the siRNA from degradation and delivered the siRNA effectively into cancer cells to target the corresponding oncogenes for gene silencing. The siRNA/dendrimer 1c complex showed considerable anticancer activity in different human cancer cell lines and in patient-derived-organoid cancer models, outperforming the current gold standard Lipofectamine 2000. In contrast to our previous dendrimers $[19,21]$, the 1c dendrimer nanosystem displayed almost no discernible cytotoxicity when tested on human kidney cells (HEK 293), mouse fibroblast cells (L929) and Madin-Darby canine kidney cells (MDCK). With effective and non-toxic delivery features, combined with simple formulation, the supramolecular dendrimer nanosystem formed by 1c holds great potential in siRNA delivery for both genomic and therapeutic applications, in particular for primary immune cells, which are very sensitive to delivery materials and therefore require especially effective and non-noxious delivery [35-37]. The present study highlights that imparting ionizable properties via tertiary amine functionalities is an effective and practical approach for siRNA delivery with a favorable safety profile. This study also offers a new perspective for a supramolecular dendrimer in biomedical applications.

\section{Acknowledgments}

This work was supported by the Ligue Nationale Contre le Cancer (LP, ZL), China Scholarship Council (WL, LD), Italian Association for Cancer Research (IG17413) (SP), the French National Research Agency under the frame of the H2020 Era-Net EURONANOMED European Research projects 'Target4Cancer', 'NANOGLIO', 'TARBRAINFECT', 'NAN-4TUM' (LP), and H2020 NMBP 'SAFE-N-MEDTECH' (LP). This article is based upon work 
from COST Action CA 17140 'Cancer Nanomedicine from the Bench to the Bedside' supported by COST (European Cooperation in Science and Technology).

\section{Author contributions}

LP coordinated the project. DD and ZL synthesized the agents; DD, LD, AT and SG characterized the agents; EL and DM performed ITC; ND and JLI provided the patient tumor models; YJ carried out cell uptake experiments; LD performed the toxicity and gel shift studies; WL performed all siRNA delivery experiments; DD, WL, LD, YJ, ZL, EL, AT, EL, NG, JLI, SG, SP and LP analyzed data; and LP wrote the paper with contributions from DD, WL and SP. All authors proofed the manuscript.

\section{Associated content}

Supporting information: Table S1, Scheme S1 and Figures S1-S8, as well as all detailed experimental methods and protocols. This information is available free of charge via the Internet.

\section{References:}

1. Setten, R. L.; Rossi, J. J.; Han, S. The Current State and Future Directions of RNAibased Therapeutics. Nat. Rev. Drug Dis. 2019, 18 (6), 421-446.

2. Delivering the Promise of RNA Therapeutics. Nat. Med. 2019, 25 (9), 1321.

3. Ledford H. Gene-silencing Technology Gets First Drug Approval After 20-year Wait. Nature. 2018, 560 (7718), 291-292.

4. Mullard, A. RNAi agents score an approval and drive an acquisition. Nat. Rev. Drug Dis. 2020, 19, 10. 
5. Kanasty, R.; Dorkin, J. R., Vegas, A., Anderson, D. Delivery Materials for siRNA Therapeutics. Nat. Mater. 2013, 12 (11), 967-77.

6. Dong, Y.; Siegwart, D. J.; Anderson, D. G. Strategies, Design, and Chemistry in siRNA Delivery Systems. Adv. Drug. Deliv. Rev. 2019, 144, 133-147.

7. Kim, B.; Park, J.; Sailor, M. J. Rekindling RNAi Therapy: Materials Design Requirements for In Vivo siRNA Delivery. Adv. Mater. 2019, 31(49), 1903637.

8. Yin, H.; Kanasty, R.; Eltoukhy, A.; Vegas, A.; Dorkin, J.; Anderson, D. Non-viral Vectors for Gene-Based Therapy. Nat. Rev. Genet. 2014, 15 (8), 541-555.

9. Ho, W.; Zhang, X.; Xu, X. Biomaterials in siRNA Delivery: A Comprehensive Review. Adv. Healthc. Mater. 2016, 5 (21), 2715-2731.

10. Rietwyk, S. Peer, D.; Next-Generation Lipids in RNA Interference Therapeutics. ACS Nano. 2017, 22 (8), 7572-7586.

11. Cooper, B. M.; Putnam, D. Polymers for siRNA Delivery: A Critical Assessment of Current Technology Prospects for Clinical Application. ACS Biomater. Sci. Eng. 2016, $2(11), 1837-1850$.

12. Khandare, J.; Calderon, M.; Dagia, N.; Haag, R. Multifunctional Dendritic Polymers in Nanomedicine: Opportunities and Challenges. Chem. Soc. Rev. 2012, 41(7), 28242848.

13. Liu, X.; Rocchi, P.; Peng, L. Dendrimers as Non-viral Vectors for siRNA Delivery. New J. Chem. 2012, 36 (2), 256-263.

14. Cao, Y.; Liu, X.; Peng, L. Molecular Engineering of Dendrimer Nanovectors for siRNA Delivery and Gene Silencing. Front. Chem. Sci. Eng. 2017, 11, 663-675.

15. Svenson, S., The dendrimer paradox - high medical expectations but poor clinical translation. Chem. Soc. Rev. 2015, 44, 4131. 
16. Dong, Y.; Yu, T.; Ding, L.; Laurini, E.; Huang, Y.; Zhang, M.; Weng, Y.; Lin, S.; Chen, P.; Marson, D.; Jiang, Y.; Giorgio, S.; Pricl, S.; Liu, X.; Rocchi, P.; Peng, L.; A Dual Targeting Dendrimer-Mediated siRNA Delivery System for Effective Gene Silencing in Cancer Therapy. J Am Chem Soc. 2018, 140 (47), 16264-16274.

17. Liu, X.; Wang, Y.; Chen, C.; Tintaru, A.; Cao, Y.; Liu, J.; Ziarelli, F.; Tang, J.; Guo, H.; Rosas, R;. Giorgio, S.; Charle, L.; Rocchi, P.; Peng, L. A Fluorinated Bolaamphiphilic Dendrimer for On-demand Delivery of siRNA, via Specific Response to Reactive Oxygen Species. Adv. Funct. Mater. 2016, 26 (47), 8594-8603.

18. Liu, X.; Zhou, J.; Yu, T.; Chen, C.; Cheng, Q.; Sengupta, K.; Huang, Y.; Li, H.; Liu, C.; Wang, Y.; Posocco, P.; Wang, M.; Cui, Q.; Giorgio, S.; Fermeglia, M.; Qu, F.; Pricl, S.; Shi, Y.; Liang, Z.; Rocchi, P.; Rossi, J.; Peng, L. Adaptive Amphiphilic Dendrimerbased Nanoassemblies as Robust and Versatile siRNA delivery Systems. Angew. Chem. Int. Ed. 2014, 53 (44), 11822-11827.

19. Yu, T.; Liu, X.; Bolcato-Bellemin, A.; Wang, Y.; Liu, C.; Erbacher, P.; Qu, F.; Rocchi P.; Behr, J.; Peng, L. An Amphiphilic Dendrimer for Effective Delivery of Small Interfering RNA and Gene Silencing in Vitro and in Vivo. Angew. Chem. Int. Ed. 2012, $51(34), 8478$.

20. Chen, C.; Posocco, P.; Liu, X.; Cheng, Q.; Laurini, E.; Zhou, J.; Liu, C.; Wang, Y.; Tang, J.; Col, V.; Yu, T.; Giorgio, S.; Fermeglia, M.; Qu, F.; Liang, Z.; Rossi, J.; Liu, M.; Rocchi, P.; Pricl, S.; Peng, L. Mastering Dendrimer Self-Assembly for Efficient siRNA Delivery: From Conceptual Design to In Vivo Efficient Gene Silencing, Small, 2016, 12 (27), 3667-3676.

21. Liu, X.; Liu, C.; Zhou, J.; Chen, C.; Qu, F.; Rossi, J.; Rocchi, P.; Peng, L. Promoting siRNA Delivery via Enhanced Cellular Uptake Using an Arginine-decorated Amphiphilic Dendrimer. Nanoscale. 2015, 7(9), 3867-75. 
22. Kulkarni, J. A.; Cullis, P. R.; Meel, R. Lipid Nanoparticles Enabling Gene Therapies: From Concepts to Clinical Utility. Nucleic Acid Ther. 2018, 28(3), 146-157.

23. Cullis, P. R., Hope, M. J., Lipid Nanoparticle Systems for Enabling Gene Therapies, Mol. Ther. 2017, 25 (7), 1467-1475.

24. Evers, M. J. W.; Kulkarni, J. A.; Meel, R.; Cullis, P. R.; Vader, P.; Schiffelers, R. M. State-of-the-Art Design and Rapid-Mixing Production Techniques of Lipid Nanoparticles for Nucleic Acid Delivery. Small Methods, 2018, 2 (9), 1700375.

25. Wu, J.; Zhou, J.; Qu, F.; Bao, P.; Zhang, Y.; Peng, L. Polycationic Dendrimers Interact With RNA Molecules: Polyamine Dendrimers Inhibit the Catalytic Activity of Candida Ribozymes, Chem. Commun. 2005, 21 (3), 313-5.

26. Manning, B. D.; Toker, A. AKT/PKB Signaling: Navigating the Network, Cell, 2017, 20(3), 381-405.

27. Franke, T. F. PI3K/Akt: Getting it Right Matters, Oncogene, 2008, 27(50), 6473-88.

28. Dang, C. V. MYC on the path to cancer, Cell, 2012, 149 (1), 22-35.

29. Chen, H.; Liu, H.; Qing, G. Targeting Oncogenic Myc as a Strategy for Cancer Treatment, Signal Transduct. Target Ther. 2018, 3, 5.

30. Tuveson, D.; Clevers, H. Cancer modelling meets human organoid technology. Science, 2019, 364 (6444), 952-955.

31. Vlachogiannis, G.; Hedayat, S.; Vatsiou, A.; Jamin, Y.; Fernández-Mateos, J.; Khan, K.; Lampis, A.; Eason, K.; Huntingford, I.; Burke, R.; Rata, M.; Koh, D.; Tunariu, N.; Collins, D.; Hulkki-Wilson, S.; Ragulan, C.; Spiteri, I.; Moorcraft, S.; Chau, I.; Rao, D. Watkins, N. Fotiadis, M. Bali, M. Darvish-Damavandi, H. Lote, Z. Eltahir, E. Smyth, Sa.; Begum, R.; Clarke, P.; Hahne, J.; Dowsett, M.; Bono, J.; Workman, P.; Sadanandam, A.; Fassan, M.; Sansom, O.; Eccles, S.; Starling, N.; Braconi, C.; Sottoriva, A.; Robinson, S.; Cunningham, D.; Valeri, N. Patient-derived Organoids 
Model Treatment Response of Metastatic Gastrointestinal Cancers, Science, 2018, 359 , 920-926.

32. Wirth, M.; Mahboobi, S.; Krämer, O. H.; Schneider, G. Concepts to Target MYC in Pancreatic Cancer, Mol Cancer Ther, 2016, 15 (8), 1792-1798.

33. Dusetti, N.; Iovanna, J. Organoids from Pancreatic Ductal Adenocarcinoma, Med Sci (Paris), 2020, 36, 57-62.

34. Bian, B.; Juiz, N.; Gayet, O.; Bigonnet, M.; Brandone, N.; Roques, J.; Cros, J.; Wang, N.; Dusetti, N.; Iovanna, J. Pancreatic Cancer Organoids for Determining Sensitivity to Bromodomain and Extra-Terminal Inhibitors (BETi), Front Oncol. 2019, 9, 475.

35. Ellert-Miklaszewska, A.; Ochocka, N.; Maleszewska, M.; Ding, L.; Laurini, E.; Jiang, Y.; Roura, A.-J.; Giorgio, S.; Gielniewski, B.; Pricl, S.; Peng, L.; Kaminska, B. Efficient and Innocuous Delivery of siRNA to Microglia Using an Amphiphilic Dendrimer Nanovector, Nanomedicine, 2019, 14, 2441-2458.

36. Garofalo, S.; Cocozza, G.; Porzia, A.; Inghilleri, M.; Raspa, M.; Scavizzi, F.; Aronica, E.; Bernardini, G.; Peng, L.; Ransohoff, R. M.; Santoni, A.; Limatola, C. Natural Killer Cells Modulate Motor Neurons-Immune Cells Cross Talk in Amyotrophic Lateral Sclerosis, Nat. Commun. 2020, 11, 1773.

37. Chen, J.; Aleksandra, E.-M.; Garofalo, S.; Dey, A.; Tang, J.; Jiang, Y.; Clément, F.; Marche, P.; Liu, X.; Kaminska, B.; Santoni, A.; Limatola, C.; Rossi, J.; Zhou, J.; Peng, L., Synthesis and use of an amphiphilic dendrimer for siRNA delivery into primary immune cells. Nat. Protoc. 2020, accepted. 